\section{Acute acquired comitant esotropia: a prospective study}

\begin{abstract}
Purpose To define the clinical characteristics of patients presenting with acute onset esotropia and features suggestive of possible underlying central nervous system pathology. To assess the prognosis for the return of binocular function and to consider the most appropriate management.

Methods A prospective clinical study was carried out of all patients presenting to the department of paediatric ophthalmology at a university teaching hospital over the period January 1994 to April 1997. Each patient underwent a full ophthalmological examination (including assessment of sensory status). All patients were referred to a paediatric neurologist for examination and CT and/or MRI scan.

Results Ten patients presented during the study period. Uncorrected hypermetropia and/ or decompensated monofixation syndrome were the commonest aetiological factors. One patient was found to have a cerebellar tumour.

implies a newly acquired deviation in a patient with some pre-existing binocularity, and should prompt careful examination for evidence of incomitance. Incomitant esotropia (due to lateral rectus paresis until proven otherwise) should be investigated appropriately in order to exclude a neuropathic or myopathic cause.

Conversely, the management of acutely acquired comitant esotropia is more controversial; although the association with central nervous system (CNS) tumour is widely recognised, ${ }^{2-11}$ it is rare, and some consider neuroradiological investigation of every case to be unnecessary. No clear guidelines exist regarding the presenting clinical features that should trigger neuroradiological investigation.

The aim of this study was to further define the characteristics of patients with acute comitant esotropia, prospectively analyse the presenting signs which may indicate underlying CNS tumour, and record the relative frequencies of each aetiology.
\end{abstract} In 5 patients prescription of the full hypermetropic correction alone was sufficient to restore binocularity. Five patients required bilateral medial rectus recession. Binocular function was restored in all cases - in 5 cases with bifoveal fusion.

Conclusion Decompensation of a pre-existing phoria or monofixation syndrome appears the commonest aetiology. Prescription of the full hypermetropic correction found at cycloplegic refraction forms an essential part of initial management. No single clinical sign can reliably indicate the rare patient harbouring a tumour. $A$ high index of suspicion should be maintained and neuro-imaging considered in the absence of expected findings such as hypermetropia or fusion potential or in the presence of atypical features or neurological signs.

Key words Acute, Comitant, Diplopia, Esotropia, Tumour

Comitant esotropia usually presents during infancy or early childhood ${ }^{1}$ and is generally considered to be a benign entity rather than a sign of underlying neurological pathology. In contrast acute onset esotropia with diplopia
CHRISTOPHER J. LYONS, PETER A.C. TIFFIN, DARREN OYSTRECK

\section{Materials and methods}

From January 1994 to April 1997 all patients presenting to the department of paediatric ophthalmology and strabismus at British Columbia's Children's Hospital with diplopia and an acute comitant esotropia were recruited into this prospective study. Each patient underwent a complete assessment including history, orthoptic testing including measurement of fusion and stereopsis on the synoptophore where possible, examination including cycloplegic refraction with $\mathrm{g}$. cyclopentolate $1 \%$, and neurological examination by a paediatric neurologist. All patients had a CT and/or MRI scan.

We prescribed spectacles to correct any refractive error and performed surgery as indicated for any persisting deviation.

Following refractive and/or surgical correction a further complete orthoptic assessment was performed with particular regard to sensory status, including measurement of stereoacuity using the Titmus test.
C.J. Lyons

P.A.C. Tiffin

D. Oystreck

Department of

Ophthalmology

British Columbia's

Children's Hospital

Vancouver, Canada

Dr C.J. Lyons

Department of

Ophthalmology

BC Children's Hospital and University of British

Columbia

Room A136, BC Children's

Hospital

4480 Oak Street

Vancouver V6H 3V4,

Canada

Tel: +1 (604) 8753117

Fax: +1 (604) 8753561

e-mail:

clyons@cw.bc.ca

Received: 23 November 1998

Accepted: 4 June 1999 


\begin{tabular}{|c|c|c|c|c|c|c|c|c|c|c|c|c|c|c|}
\hline \multirow{2}{*}{$\begin{array}{l}\text { Patient } \\
\text { no. }\end{array}$} & \multirow{2}{*}{$\begin{array}{c}\text { Age } \\
\text { (years) }\end{array}$} & \multirow{2}{*}{$\begin{array}{l}\text { Duration } \\
\text { (months) }\end{array}$} & \multirow[b]{2}{*}{ Symptom } & \multirow{2}{*}{$\begin{array}{l}\text { Family } \\
\text { history }\end{array}$} & \multirow[b]{2}{*}{ Nystagmus } & \multicolumn{2}{|c|}{$\begin{array}{l}\text { Deviation } \\
\text { (dioptres) }\end{array}$} & \multirow{2}{*}{$\begin{array}{l}\text { Fusion } \\
\text { before } \\
\text { therapy }\end{array}$} & \multirow[b]{2}{*}{ Refraction } & \multirow{2}{*}{$\begin{array}{l}\text { Neurologi- } \\
\text { cal exam. }\end{array}$} & \multirow[b]{2}{*}{ Scan } & \multirow[b]{2}{*}{ Treatment } & \multirow[b]{2}{*}{ Outcome } & \multirow{2}{*}{$\begin{array}{l}\text { Follow-up } \\
\text { (months) }\end{array}$} \\
\hline & & & & & & $\mathrm{D}$ & $\mathrm{N}$ & & & & & & & \\
\hline 1 & 5 & 5 & DV & & & 35 & 60 & Central & $\begin{array}{l}+3.75 \\
+3.75\end{array}$ & $\mathrm{~N}$ & $\mathrm{~N}$ & gls & $60 \mathrm{~s} . \mathrm{F}$ & 16 \\
\hline 2 & 10 & 8 & DV & + & & 30 & 30 & Central & $\begin{array}{l}+2.25 \\
+1.50\end{array}$ & $\mathrm{~N}$ & $\mathrm{~N}$ & g. $5 x$ & 40s.F & 27 \\
\hline 3 & 3.5 & 1 & C & & + & 25 & 20 & Peripheral & $\begin{array}{l}+2.50 \\
+2.50\end{array}$ & $\mathrm{~N}$ & $\mathrm{~N}$ & gls & MF & 18 \\
\hline 4 & 4 & 1 & DV & & & 25 & 25 & L suppression & $\begin{array}{l}+2.75 \\
+1.50\end{array}$ & $\mathrm{~N}$ & $\mathrm{~N}$ & gls & $\mathrm{MF}$ & 20 \\
\hline 5 & 5 & 5 & DV & + & & 35 & 40 & L suppression & $\begin{array}{l}+4.00 \\
+3.50\end{array}$ & $\mathrm{~N}$ & $\mathrm{~N}$ & g. $S x$ & $40 \mathrm{sF}$ & 34 \\
\hline 6 & 24 & 12 & DV & + & + & 20 & 30 & Central & $\begin{array}{l}+5.75 \\
+4.75\end{array}$ & $\mathrm{~N}$ & $\mathrm{~N}$ & g Sx & MF & 33 \\
\hline 7 & 7.5 & 2 & C & + & + & 50 & 60 & $\mathrm{R}$ suppression & $\begin{array}{l}+1.50 \\
+1.50\end{array}$ & $\mathrm{~N}$ & $\mathrm{~N}$ & g. $5 x$ & $60 \mathrm{~s} ? \mathrm{~F}$ & 24 \\
\hline 8 & 6 & 12 & C & & & 25 & 25 & Central & $\begin{array}{l}+1.50 \\
+1.25\end{array}$ & $\mathrm{~N}$ & $\mathrm{~N}$ & gls & MF & 10 \\
\hline 9 & 4.5 & 2.5 & C & & & 35 & 35 & L suppression & $\begin{array}{l}0.00 \\
0.00\end{array}$ & $\mathbf{N}$ & $\mathbf{T}$ & Sx & 40s.F & 24 \\
\hline 10 & 5 & 6 & DV & & & 20 & 20 & Central & $\begin{array}{l}+2.25 \\
+2.25\end{array}$ & $\mathrm{~N}$ & $\mathrm{~N}$ & gls & MF & 12 \\
\hline
\end{tabular}

DV, double vision; C, covers one eye; D, distance; N, near; Central, fusion of central slides on synoptophore; Peripheral, fusion of peripheral slides on synoptophore; N, normal; T, tumour; gls, glasses only; g. Sx, glasses and strabismus surgery; Sx, surgery only; 40s, 60s, stereoacuity on Titmus test; F, bifoveal fusion; MF, monofixation syndrome.

\section{Results}

Ten patients presented during the study period (Table 1). There were 5 males and 5 females. Their ages ranged from 3.5 to 24 years (median 5 years). Surprisingly some patients had been symptomatic for up to 12 months before presentation. Total follow-up from onset of symptoms ranged from 10 to 34 months (mean 21.8 months). Six patients spontaneously complained of seeing double, the youngest to do so being 4 years old. The other 4 were noted to close one eye constantly and reported double vision when asked. Four patients had a family history of strabismus. Interestingly patient 7 had a sister who had also previously presented elsewhere with an acute comitant esotropia at age 4 years.

One of the 10 patients was found to have an underlying tumour (patient 9). This 41/2-year-old girl presented with a 10 week history of covering one eye and double vision which at first had been intermittent. For the previous month her parents had noticed that her left eye continuously turned in. Examination revealed a 35 PD esotropia which was comitant on side gazes. There was an associated V-pattern with an increase from 25 PD in upgaze to 45 PD on downgaze. There was no abducting nystagmus or lateral rectus weakness and no fusion was demonstrable on the synoptophore. Her optic discs appeared normal. Unlike the rest of the group, she had no refractive error. Also, she had no family history of strabismus. She had a normal neurological examination with, surprisingly, apparently normal cerebellar function. CT and MRI (Fig. 1) showed a very large, midline, gadolinium-enhancing cerebellar tumour. This was excised surgically and proved to be a grade I astrocytoma. Despite total excision, her strabismus was unchanged. Both medial recti were recessed $5 \mathrm{~mm}$ 3 months after the onset of esotropia, resulting in restoration of bifoveal fusion with 40 seconds of arc stereopsis on the Titmus test.
All the other 9 patients were hypermetropic and were initially prescribed their full optical correction. In 5 cases this was sufficient to restore ocular alignment and fusion (as demonstrated by the presence of stereopsis using the Titmus test), while 4 others subsequently required bilateral medial rectus recessions.

In all cases sensory testing after treatment indicated restoration of binocularity. Five patients appeared to have regained bifoveal fusion and 5 showed features compatible with a monofixation syndrome. These findings have remained stable throughout the period of follow-up, with no patient demonstrating a recurrence of symptoms or requiring further surgery.

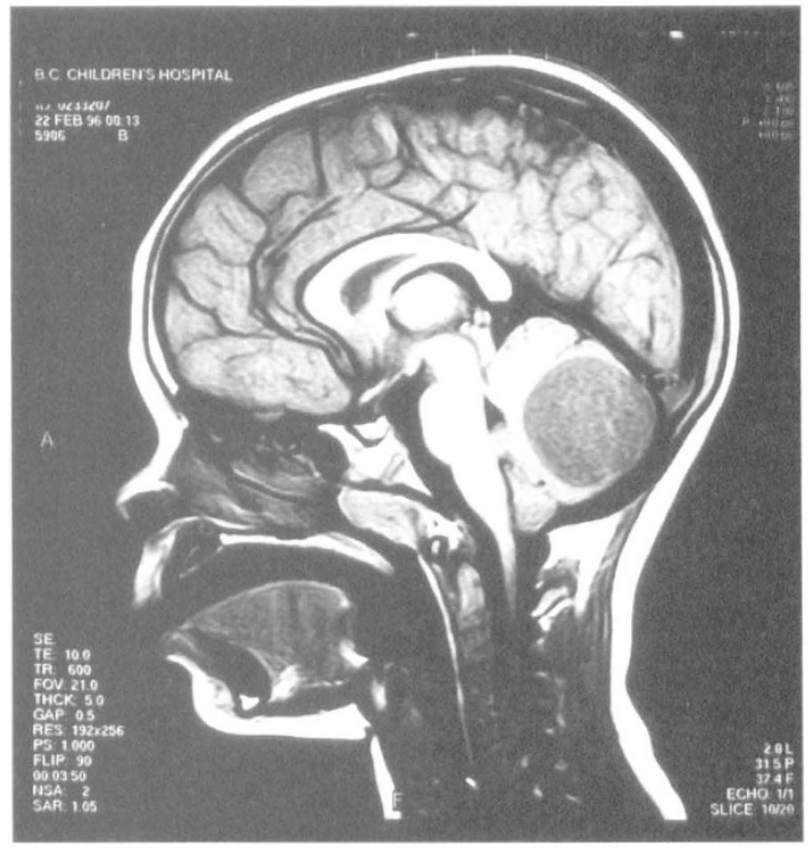

Fig. 1. MRI scan of a 4-year-old girl (patient 9) with a comitant 35 PD esotropia and no abducting nystagmus or demonstrable fusion. Neurological examination was normal. MRI scan demonstrates a large midline cerebellar tumour with gadolinium enhancement. 


\section{Discussion}

Hoyt and Good ${ }^{10}$ after Burian and Miller ${ }^{12}$ have proposed three distinct categories for acute onset comitant esotropia based on clinical features and aetiology. All three categories have a large angle of deviation, good binocular potential and no underlying neurological pathology. Type I ('Swan type'13) follows disruption of binocularity by monocular occlusion, type II ('Franceschetti type ${ }^{\prime 14}$ ) has no obvious underlying cause other than possible 'physical or psychic shock' and type III ('Bielschowsky type ${ }^{\prime 15}$ ) is associated with myopia.

Our study confirms acquired comitant esotropia to be a rare condition, with only 10 cases presenting to our department over a three and a half year period. Of these, only one patient's findings (patient 7) were compatible with a type II ('Franceschetti type') category. This patient's elder sister had a similar history, a familial tendency which has been previously reported both for siblings ${ }^{16}$ and monozygous twins. ${ }^{17}$ In 8 of our 10 patients uncorrected hypermetropia and/or decompensated monofixation syndrome were thought to be the significant aetiological factor. Only one patient was found to have a tumour. Legmann Simon et al. ${ }^{18}$ found a significant refractive element in 7 of 10 patients presenting over a 10-year period. Clark et al. ${ }^{19}$ reported 6 children with acute acquired non-accommodative esotropia all of whom had a normal neurological examination and normal CT scan.

In previous reports the angle of deviation in acute esotropia has been larger than in some of the patients in this series. For example, the mean near angle at presentation was 54 PD in a recently reported group of patients, ${ }^{18}$ although a wide variation was noted. Our strict criterion of double vision as a presenting feature could account for this variation from the norm, since a new tropia of any angle might be expected to be symptomatic in a previously binocular patient.

We chose to include patients presenting with acquired comitant esotropia and symptoms compatible with diplopia in our series. Although younger children often do not describe double vision they may manifest their adaptations to this, such as closing or covering one eye. Our youngest patient to complain of seeing double was 4 years old. Very young children adapt rapidly to comitant strabismus by suppressing the deviating eye; acute comitant esotropia due to cerebellar astrocytoma has been reported in two children aged only 18 and 27 months. ${ }^{9}$ Clearly, the presence of diplopia is insufficient to identify every child with a tumour, but its presence indicates recent change such as development of new manifest strabismus, or decompensation of a previous monofixation pattern.

Underlying intracranial neoplasia is well documented as a cause of acute comitant estropia. Tumours of the cerebellum, pons, corpus callosum and hypothalamic region have all been implicated. ${ }^{2-11}$ However, the exact mechanism(s) by which these varied tumours may produce a comitant deviation is not clear. Flynn ${ }^{3}$ described the case of an 11-year-old girl who initially presented with a comitant esotropia but subsequently developed signs of facial weakness and other lower cranial nerve dysfunction secondary to a pontine glioma. The likely aetiology of the comitant esotropia was early sixth nerve paresis; in discussing the case Jampolsky ${ }^{20}$ commented that the finding of a small V-pattern at presentation could suggest the possibility of primary sixth nerve paresis. In this regard it is interesting that our patient with a midline cerebellar tumour also displayed a $\mathrm{V}$-pattern without other demonstrable signs of lateral rectus weakness.

Hydrocephalus is an occasional cause of comitant esotropia. ${ }^{21}$ Acute comitant strabismus has also been well documented in patients with intracranial tumour but no hydrocephalus. Only 2 of the 6 patients described by Williams and $\mathrm{Hoyt}^{4}$ had elevated intracranial pressure. In these cases one might postulate a direct effect on the brainstem or cerebellum resulting in the development of a comitant deviation. Ciancia $^{7}$ discussed a series of 8 patients with posterior fossa tumour and comitant esotropia. Seven had abducting nystagmus and 6 had asymmetric optokinetic nystagmus. Lennerstrand et $a .^{22}$ found a strong correlation between comitant strabismus and hydrocephalus in patients with myelomeningocele. Convergence defects were also found to correlate with deformities of the tectal plate. They suggested that strabismus in these patients may therefore either relate to general CNS dysfunction in association with hydrocephalus or even that enlargement of the third ventricle could directly affect oculomotor centres (particularly vergence control areas) in the upper brainstem. Hoyt and Good ${ }^{10}$ find the concept of direct involvement of vergence centres in the mesencephalon an attractive hypothesis. They point out that pure 'vergence' cells have been identified in the primate midbrain. ${ }^{23}$ In addition Gomez et al. ${ }^{24}$ have suggested involvement of vergence control systems as a possible cause for the acute monocular esotropia described in 3 patients with posterior thalamic infarctions associated with signs of rostral midbrain dysfunction. Further evidence for an active divergence centre is also provided by the finding of normal saccadic velocities in patients with divergence paralysis esotropia, ${ }^{25}$ implying this to be a distinct clinical entity unrelated to sixth nerve paresis.

Clinically, the presence of abducting nystagmus, failure to re-establish binocularity after ocular realignment, evidence of a V-pattern or slightly greater deviation in the distance have all been cited as clinical signs which should raise the index of suspicion for underlying CNS tumour in patients with acute comitant esotropia. ${ }^{4,10,20,26}$

Abducting nystagmus was a presenting feature in 3 patients in our series, none of whom had serious intracranial pathology. It was absent in the patient with cerebellar tumour. The presence of abducting nystagmus in previous patients with documented intracranial tumours could represent fixation duress in abduction in patients with early, otherwise subclinical sixth nerve palsy. 
In this series the pre-treatment fusional status of the patient as assessed on the synoptophore was not predictive for either the presence of tumour or the eventual degree of binocular function obtained after treatment. Two of the patients with suppression at the time of presentation (including patient 9) subsequently regained bifoveal fusion with 40 seconds of stereopsis on the Titmus test. However, the cerebellar tumour of patient 9 had been removed by the time the eyes were realigned, in contrast to previous retrospective reports on the subject where the failure to achieve post-operative fusion was an indicator of the presence of residual intracranial tumour. Binocularity was restored in all patients after treatment: a monofixation pattern was present in 5, and bifoveal fusion in 5 others. These good results may reflect the short time which elapsed between diagnosis and correction of the strabismus through refractive or surgical means.

Our study shows that no single clinical sign reliably indicates which patient with acute esotropia is harbouring a CNS tumour; none of our 3 patients with abducting nystagmus and only 1 of 4 patients with loss of fusion on the synoptophore at presentation had a tumour. However, this patient was also the only one to demonstrate a significant V-pattern and absence of hypermetropia. Lack of any other 'risk factors' for strabismus, such as a positive family history or hypermetropia, should raise the index of suspicion for the presence of tumour.

In conclusion, this is the first prospective series in which patients have been recruited on the basis of presentation with symptoms or signs compatible with double vision, and clinical findings of comitant esotropia. It confirms that, rather than the Swan, Franceschetti or Bielschowsky types of comitant deviation, decompensation of a pre-existing phoria or monofixation syndrome is the most common cause of this presentation. Prescription of the full hypermetropic error found at cycloplegic refraction is essential. Although underlying tumour or other neurological disease is rare (probably accounting for much less than $10 \%$ of cases) a high index of suspicion should be maintained. Unfortunately no particular finding, including age, is entirely discriminatory in this regard.

Neuroradiological investigation should be prompted by the presence of associated neurological symptoms such as headache or unsteadiness or signs such as abduction nystagmus which may indicate an incipient sixth nerve palsy, or the absence of the findings expected to accompany 'benign' acquired comitant strabismus such as hypermetropia, family history or loss of fusion. This study confirms the wide 'grey area' surrounding this presentation and emphasises the need for a clinical algorithm which is based on caution when managing these patients.

\section{References}

1. Burian-von Noorden GK. Binocular vision and ocular motility. St Louis: CV Mosby, 1990:389-90.

2. Anderson DW, Lubow M. Astrocytoma of the corpus callosum presenting with acute comitant esotropia. Am J Ophthalmol 1970;69:594-8.

3. Flynn JT. Problems in strabismus management. Transactions of the New Orleans Academy of Ophthalmology. New York: Raven Press, 1986:456.

4. Williams AS, Hoyt CS. Acute comitant esotropia in children with brain tumors. Arch Ophthalmol 1989;107:376-8.

5. DeYoung Smith M, Baker JD. Esotropia as the presenting sign of brain tumor. Am Orthopt J 1990;40:72-5.

6. Astle WF, Miller SJ. Acute comitant estropia: a sign of intracranial disease. Can J Ophthalmol 1994;29:151-4.

7. Ciancia AO. On infantile esotropia with nystagmus in abduction. J Pediatr Ophthalmol Strabismus 1995;32:280-8.

8. Timms C, Taylor D. Intracranial pathology in children presenting as comitant strabismus. In: Update on strabismus and pediatric ophthalmology. Boca Raton, FL: CRC Press, 1995:593-6.

9. Jaafar MS, Collins MLZ, Rabinowitz AI. Cerebellar astrocytoma presenting as acquired comitant esotropia at age 18 and 27 months. In: Update on strabismus and pediatric ophthalmology. Boca Raton, FL: CRC Press, 1995:597-600.

10. Hoyt CS, Good WV. Acute onset concomitant esotropia: when is it a sign of serious neurological disease? Br J Ophthalmol 1995;79:498-501.

11. Simon JW, Waldman JB, Couture KC. Cerebellar astrocytoma manifesting as isolated, comitant esotropia in childhood. Am J Ophthalmol 1996;121:584-6.

12. Burian HM, Miller JE. Comitant convergent strabismus with acute onset. Am J Ophthalmol 1958;45:55-63.

13. Swan KC. Esotropia following occlusion. Arch Ophthalmol 1947;36:444-51.

14. Franceschetti A. Le strabise concomitant aigu. Ophthalmologica 1952;123:219-26.

15. Bielschowsky A. Das Einwartfschien der Myopia. Ophthalmol Ges 1892;43:245-8.

16. Norbis AL, Malbran E. Concomitant esotropia of late onset: pathological report in four cases in siblings. Br J Ophthalmol 1956;40:373-8.

17. Ahmed S, Young JDH. Late onset esotropia in monozygous twins. Br J Ophthalmol 1993;77:189-91.

18. Legmann Simon A, Borchert M. Etiology and prognosis of acute, late-onset estropia. Ophthalmology 1997;104:1348-52.

19. Clark AC, Nelson LB, Simon JW, et al. Acute acquired comitant esotropia. Br J Ophthalmol 1989;73:636-8.

20. Jampolsky AJ. Problems in strabismus management. Transactions of the New Orleans Academy of Ophthalmology. New York: Raven Press, 1986:456.

21. Harcourt RB. Ophthalmic complications of meningomyelocele and hydrocephalus in children. $\mathrm{Br} \mathrm{J}$ Ophthalmol 1968;52:670-6.

22. Lennerstrand G, Gallo JE, Samuelsson L. Neuroophthalmological findings in relation to CNS lesions in patients with myelomeningocele. Dev Med Child Neurol 1990;32:423-31.

23. Judge J, Cummings BG. Neurons in the monkey midbrain with activity related to divergence eye movements and accommodation. J Neurophysiol 1986;55:915-30.

24. Gomez CR, Gomez SM, Selhorst JB. Acute thalamic esotropia. Neurology 1988;38:1759-6.

25. Lim L, Rosenbaum AL, Demer JL. Saccadic velocity analysis in patients with divergence paralysis. J Pediatr Ophthalmol Strabismus 1995;32:76-81.

26. Scott W. Problems in strabismus management. Transactions of the New Orleans Academy of Ophthalmology. New York: Raven Press, 1986:456. 